\title{
Two potential biomarkers identified in mesenchymal stem cells and leukocytes of patients with sporadic amyotrophic lateral sclerosis
}

\author{
Henny Nachmany ${ }^{\mathrm{a}, 1}$, Shane Wald ${ }^{\mathrm{a}, 1}$, Michal Abekasis ${ }^{\mathrm{a}}$, Shlomo Bulvik ${ }^{\mathrm{b}}$ and Miguel Weil ${ }^{\mathrm{a}, *}$ \\ ${ }^{a}$ Laboratory for Neurodegenerative Diseases and Personalized Medicine, Department of Cell Research and \\ Immunology, The George S. Wise Faculty of Life Sciences, Tel Aviv University, Tel Aviv. Israel \\ ${ }^{\mathrm{b}}$ Hematology Department, Laniado Hospital, Netanya, Israel
}

\begin{abstract}
Amyotrophic lateral sclerosis (ALS) is a fatal, neurodegenerative disorder caused by degeneration of motor neurons. The cause for most cases of ALS is multi-factorial,this enhances the need to characterize and isolate specific biomarkers found in biological samples from ALS patients. To this end we use human mesenchymal stem cells (hMSC) derived from the bone marrow of six ALS patients (ALS hMSC) and identified two genes, Cytoplasmic FMR Interacting Protein 2 (CyFIP2) and Retinoblastoma $(\mathrm{Rb})$ Binding Protein 9 (RbBP9) with a significant decrease in post transcriptional A to I RNA editing compared to hMSC of healthy individuals. At the transcriptional level we show abnormal expression of these two genes in ALS hMSC by quantitative real time-PCR (qRT-PCR) and Western blot suggesting a problem in the regulation of these genes in ALS. To strengthen this view we tested by qRT-PCR the expression of these genes in peripheral blood leukocytes (PBL) isolated from blood samples of 17 ALS patients and found that CyFIP2 and RbBP9 levels of expression were significantly different compared to the levels of expression of these two genes in 19 normal PBL samples. Altogether we found two novel ALS potential biomarkers in non-neural tissues from ALS patients that may have direct diagnostic and therapeutic implications to the disease.
\end{abstract}

Keywords: ALS biomarkers, CyFIP2, RbBP9, human mesenchymal stem cells, leukocytes

\section{Background}

ALS is a fatal and incurable neurodegenerative disorder characterized by progressive loss of motor neurons in the spinal cord, brainstem and motor cortex, resulting in generalized weakness and muscle atrophy. ALS is the most common motor neuron disorder, with a prevalence of 6 per 100,000 at any given time [1]. Approximately $90 \%$ of the cases are sporadic, and the remaining $10 \%$ are familial [2]. Over 100 distinct mu-

\footnotetext{
${ }^{1}$ Equal contribution to this work.

*Corresponding author: Miguel Weil, Department of Cell Research and Immunology, The George S. Wise Faculty of Life Sciences, Tel Aviv University Ramat Aviv 69978, Tel Aviv, Israel. Tel.: +972 3 6406981; Fax: +972 3 6422046; E-mail: miguelw@ tauex.tau.ac.il.
}

tations in the ubiquitously expressed enzyme $\mathrm{Cu} / \mathrm{Zn}$ superoxide dismutase-1 (SOD1) have been identified in approximately $20 \%$ of familial cases of ALS [3,4]. The striking pathological and clinical similarity between familial and sporadic disease has sparked enthusiasm that the animal models based on mutant SOD1 might provide insight into mechanisms of both sporadic and familial disease. The causes for most cases of ALS are unknown and the clinical course is highly variable, suggesting that multiple factors underlie the disease mechanism [5]. This fact enhances the need to use a multidisciplinary approach to characterize and isolate specific biomarkers found in biological samples from ALS patients. To this end we use human mesenchymal stem cells (hMSC) derivedfrom the bone marrow of ALS patients and characterize their biological potential to identify ALS related biomarkers. Bone marrow con- 
tains two major populations of stem cells: hematopoietic stem cells and mesenchymal stem cells occasionally referred to as bone marrow stromal cells. These cells can differentiate into cells of mesenchymal lineage such as osteoblasts, chondrocytes and adipocytes [6]. Moreover, previous study in our laboratory reveals that these stem cells bearthe rare biological capacity to survive for prolonged time under serum free culture conditions due to the production of their own survival factors, like bone morphogenetic protein [7].

RNA editing is a post-transcriptional mechanism for expanding the proteomic repertoire. Adenosineto-inosine (A to I) RNA editing by enzymes named ADARs (adenosine deaminases acting on RNAs) is essential for normal life and development of both invertebrates and vertebrates [8-10]. When functioning improperly, this essential process, can lead to pathological conditions ranging from epilepsy to malignant gliomas [11-13]. So far, only abnormal RNA editing at the $\mathrm{Q} / \mathrm{R}$ (glutamine/arginine) site of the GluR2 gene of the AMPA (Amino-3-hydroxy-5-methyl-4isoxazolepropionate) receptor has been identified to be implicated in ALS [14-17]. It is unknown whether the RNA editing deficiency in the GluR-2 gene is part of a more global RNA problem related with ADARs activity in ALS [18].

Here we show that ALS hMSC have abnormal pattern of RNA editing in two novel genes, CyFIP2 and RbBP9, which also expressed significantly different in hMSC and PBL of ALS patients compared with samples from normal individuals. Overall, we show that non-neural samples of ALS patients could serve as model tools to reveal potential biomarkers of the disease that could be used for future direct diagnosis of ALS in blood samples.

\section{Results}

hMSC were isolated from bone marrow samples of 6 ALS patients (ALS hMSC) and from 6 non-ALS donors, based on their known property to adhere onto a plastic surface [19]. These isolated cells were routinely characterized by the expression pattern of a number of known surface markers such as $\mathrm{CD}_{105^{+}}, \mathrm{CD} 90^{+}$, $\mathrm{CD}^{+} 3^{+}, \mathrm{CD}^{-} 5^{-}, \mathrm{CD}_{19}^{-}$and $\mathrm{CD}^{-} 4^{-}$measured by FACS (Fluorescence-activated cell sorting) and by their ability to differentiate into adipocytes, osteocytes and chondrocytes, as established by The International Society for Cellular Therapy [20]. Moreover, as mentioned above we also characterized hMSC by their biological property of prolonged survival under serum free conditions [7,21]. To this end ALS hMSC and non-ALS hMSC were cultured at low and high densities of 20 and 60 cells per square millimeter in 24 well dishes, respectively in DMEM with or without $10 \%$ serum. Cell viability in each well was determined at the end of the incubation period of 1,2 and 3 days using a vital fluorescent dye mix containing Calcein-AM (stains living cells), Propidium iodide (PI) (stains dead cells) and Hoechst 33342 (stains all nuclei) as described previously [7]. Figure 1 shows representative results from these characterization experiments of hMSC. Overall, the biological features in ALS hMSC, surface markers expression (Fig. 1A), cell survival (Fig. 1B) and differentiation capacity (Fig. 1C), are indistinguishable from non-ALS hMSC.

From these common biological features between the hMSC donors we tested the hypothesis that ALS hMSC may serve as a model tools for ALS biomarker identification. We investigated in ALS hMSC the possibility that A to I conversion by RNA editing may be altered in specific target substrate genes of the ADAR-mediated RNA editing enzymes [22], as previously observed in ALS [14]. We analyzed RNA editing levels in ALS and non-ALS hMSC of 10 target genes that are known to undergo A to I RNA editing: FANCC, BLCAP, BRCA1, CyFIP2, RbBP9, MDM4, FLNA, GluR-2 (the Q/R site), CARD11 and [22]. RNA editing analysis was performed on cDNA synthesized by reverse transcription of RNA extracts from ALS and non-ALS hMSC samples that were cultured with or without serum for 5 days. Figure 2A shows the results of this analysis, where only two out of the ten genes tested, CyFIP2 and RbBP9, appeared to have A to I RNA editing levels that are significantly reduced in all ALS hMSC samples when compared to the samples of hMSC of non-ALS individuals.

To verify these results we performed RT-PCR analysis on the same RNA samples from 6 ALS and 3 nonALS hMSC with the addition of 3 new non-ALS hMSC samples as we show in Fig. 2B. The results show that CyFIP2 and RbBP9 genes are constitutively expressed in hMSC. Therefore we confirm that the decrease in RNA editing in ALS hMSC samples in Fig. 2A is due to RNA editing deficiency and not due to the lack of RNA transcription of these genes.

These results lead us to investigate the mRNA expression levels of CyFIP2 and RbBP9 genes in ALS and non-ALS hMSC cultures using qRT-PCR analysis. In these experiments we found significant expression differences for these genes between ALS and non-ALS 
A

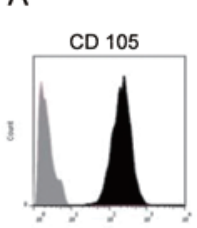

$\mathrm{CD} 34$

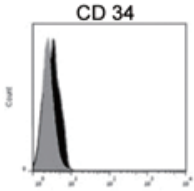

B
Non-ALS hMSC

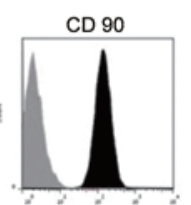

CD 45

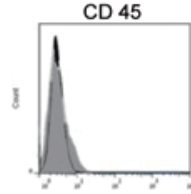

Non-ALS hMSC
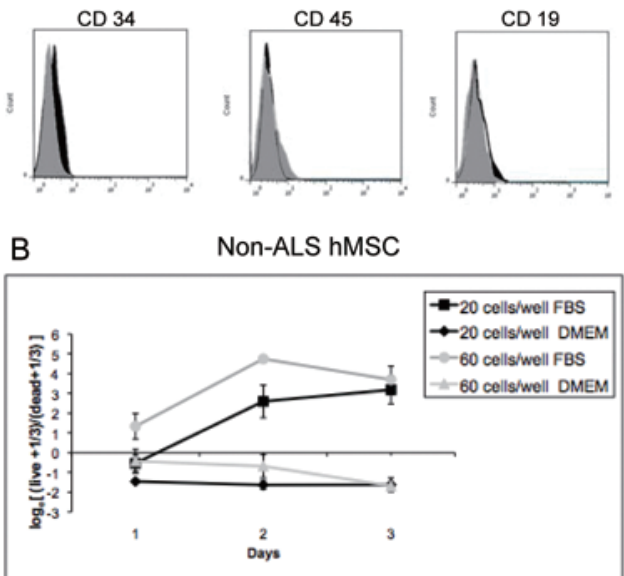

ALS hMSC

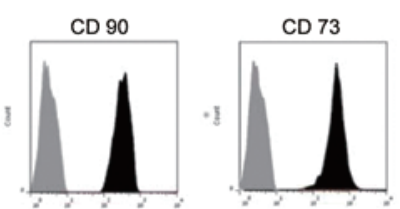

$\mathrm{CD} 34$
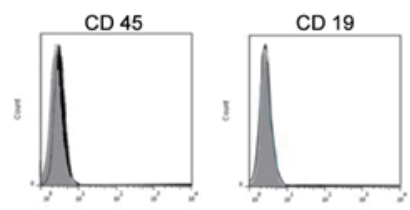

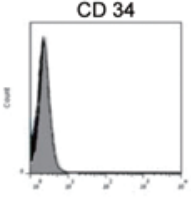

ALS hMSC

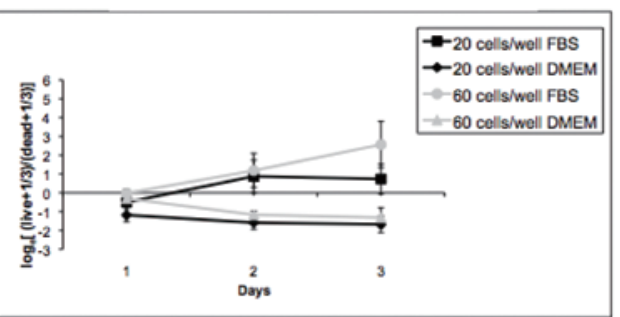

ALS hMSC

C

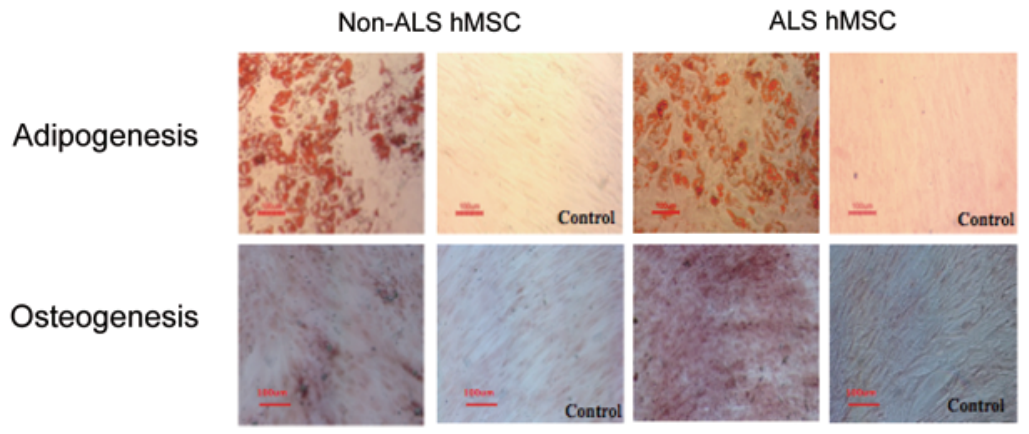

Fig. 1. Representative characterization of hMSC from ALS and non-ALS samples. A. FACS analysis of hMSC surface markers: CD105 ${ }^{+}$, $\mathrm{CD}^{+} 0^{+}, \mathrm{CD} 3^{+}, \mathrm{CD}^{-} 5^{-}, \mathrm{CD} 19^{-}, \mathrm{CD} 34^{-}$. B. Comparative analysis of cell survival of ALS hMSC $(n=4)$ and non-ALS $(n=2)$ donors at two low densities cultured for up to 3 days in the presence or absence of serum. For comparative analysis between different samples and experiments the mean values are expressed as Log of the live/dead ratio as indicated in y axis. The conditions were scored in triplicates of two independent experiments. C. ALS and non-ALS hMSC differentiate into adipocytes and osteoblasts. Cells were grown either in adipogenesis, or osteogenesis differentiation medium or in control medium (DMEM $+10 \%$ FCS) for 21 days. The cells were fixed and stained specifically to detect differentiated cells with oil red for adipocytes and Alizarin red for osteoblasts. Note that control cells show typical hMSC morphologies and did not stain with the differentiation specific dyes.

hMSC as shown in Fig. 3. Following these experiments we examined the protein expression levels of these two genes by Western blot analysis of ALS and non-ALS hMSC protein extracts. Figure 4 shows that the protein levels of CyFIP2 and RbBP9 are significantly low in all ALS hMSC tested compared to non-ALS hMSC.

Overall, the ALS specific RNA editing pattern, found consistently in all ALS individuals for CyFIP2 and RbBP9, and the differences found at the transcriptional and translational levels of these genes, strongly support our view that ALS hMSC are useful model tool to identify possible ALS biomarkers. To strengthen the possibility that these genes could become future diagnostic ALS biomarkers, we performed several experiments on PBL samples from ALS and non-ALS individuals. After verification of the expression of these genes in ALS and non-ALS PBL samples we performed RNA editing analysis on RNA samples and found that both genes, CyFIP2 and RbBP9 are not RNA edited in PBL while the ADAR1 editing control gene BLCAP (bladder cancer associated protein) [23] showed similar RNA editing levels in all PBL samples (data not shown). Having determined that CyFIP2 and RbBP9 are not post-transcriptionally modified by A to I RNA 
A
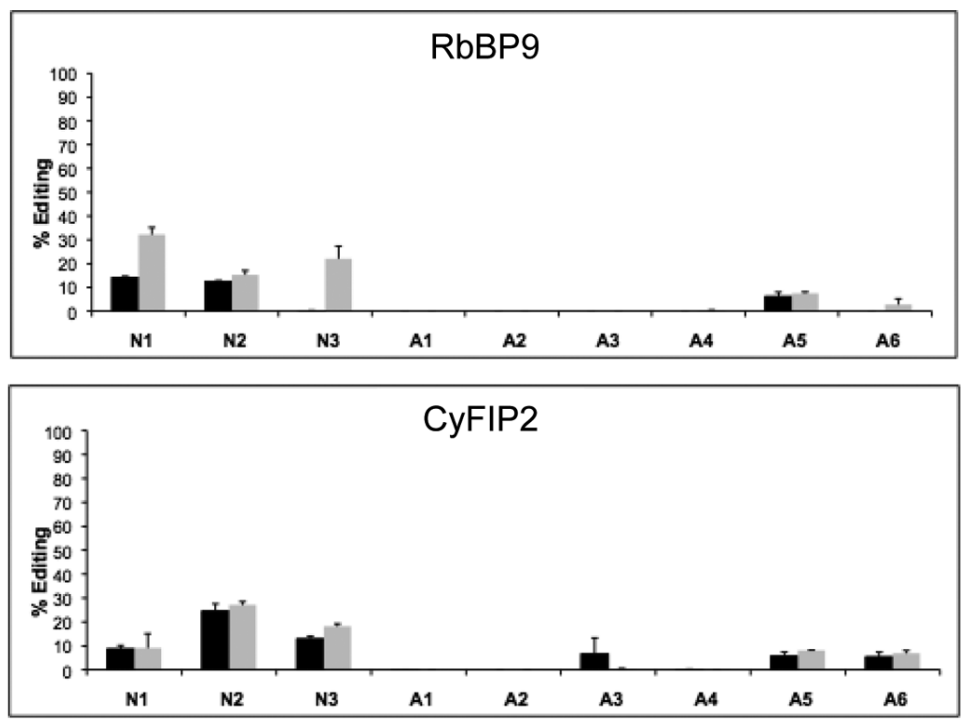

B

Non-ALS hMSC

ALS hMSC

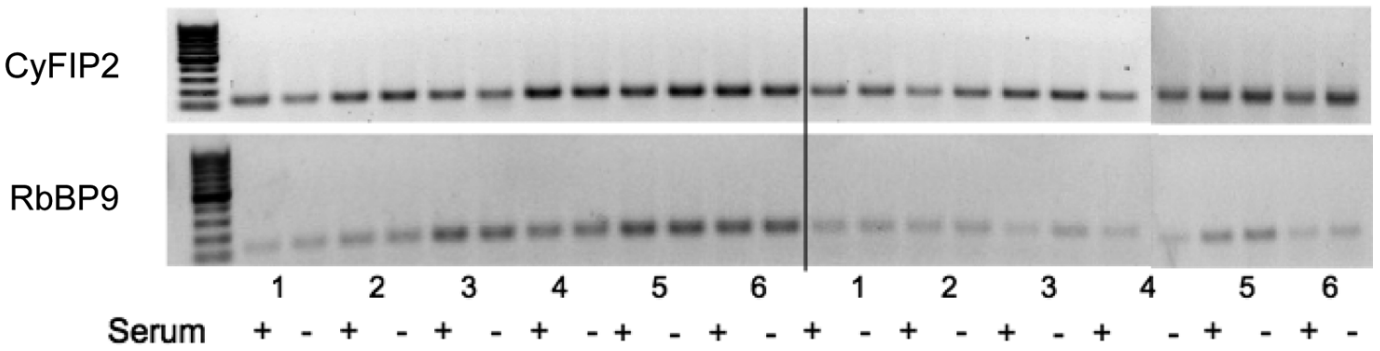

Fig. 2. mRNA editing levels of CyFIP2 and RbBP9 in ALS and non-ALS hMSC. A. RNA extracts were prepared from ALS ( $n=6$; A1-6) and non-ALS ( $n=3$; N1-3) hMSC at passages 3-4 that were grown in 10\% FBS or DMEM alone for 5 days. cDNA was produced by in vitro reverse transcription for the RNA editing analysis. Editing efficiency of these genes was measured in the cDNA samples as \% of total editing and represent as mean $+/-$ standard deviation (s.d). Statistical analysis of the significant difference between ALS and non-ALS groups for the two genes under the two different culture conditions was performed by T-test after square root transformation. In DMEM the significance for the two genes was: CyFIP2 $p=0.01 ; \operatorname{RbBP} 9 p=0.001$. In FBS the significance was only for CyFIP2 $p=0.019$. B. Shows RT-PCR results of the expression of CyFIP2 and RbBP9 in RNA extracts of the same 6 ALS hMSC and 6 non-ALS hMSC to confirm the expression of both genes in the samples that were analyzed in A (with the addition of three new non-ALS hMSC grown at the same culture conditions). The specificity of the PCR products was confirmed by DNA sequencing.

editing in PBL, we wanted to investigate whether these genes are differentially expressed in ALS PBL as shown above in hMSC (see Fig. 2). To do so, we performed qRT-PCR experiments to determine the mRNA expression levels of CyFIP2 and RbBP9 in PBL isolates from blood samples of a total of 17 ALS and 19 non-ALS individuals. Figure 5 shows the results from these experiments were we detected a significant difference in mRNA expression levels of both genes in ALS compared to non-ALS PBL samples. These results found in ALS-PBL may support that the genes CyFIP2 and RbBP9, which are also miss-expressed in ALS hMSC, could serve as diagnostic biomarker tools for detection of ALS in blood samples.

\section{Discussion}

Here we have identified two genes CyFIP2 and $\mathrm{RbBP} 9$ that are differentially expressed in bone marrow derived hMSC and in PBL of ALS patients. Interestingly, these genes were isolated from 10 RNA editing target genes by their different A to I RNA editing pattern found in all six samples of ALS hMSC after being kept for several passages in culture. It is still unknown what functional effect does the lack of A to I conversion of RNA editing may have on CyFIP2 and RbBP9 proteins and how this may affect ALS hMSC biology. Moreover, we also don't know the meaning of the difference in the transcriptional levels of gene expression 


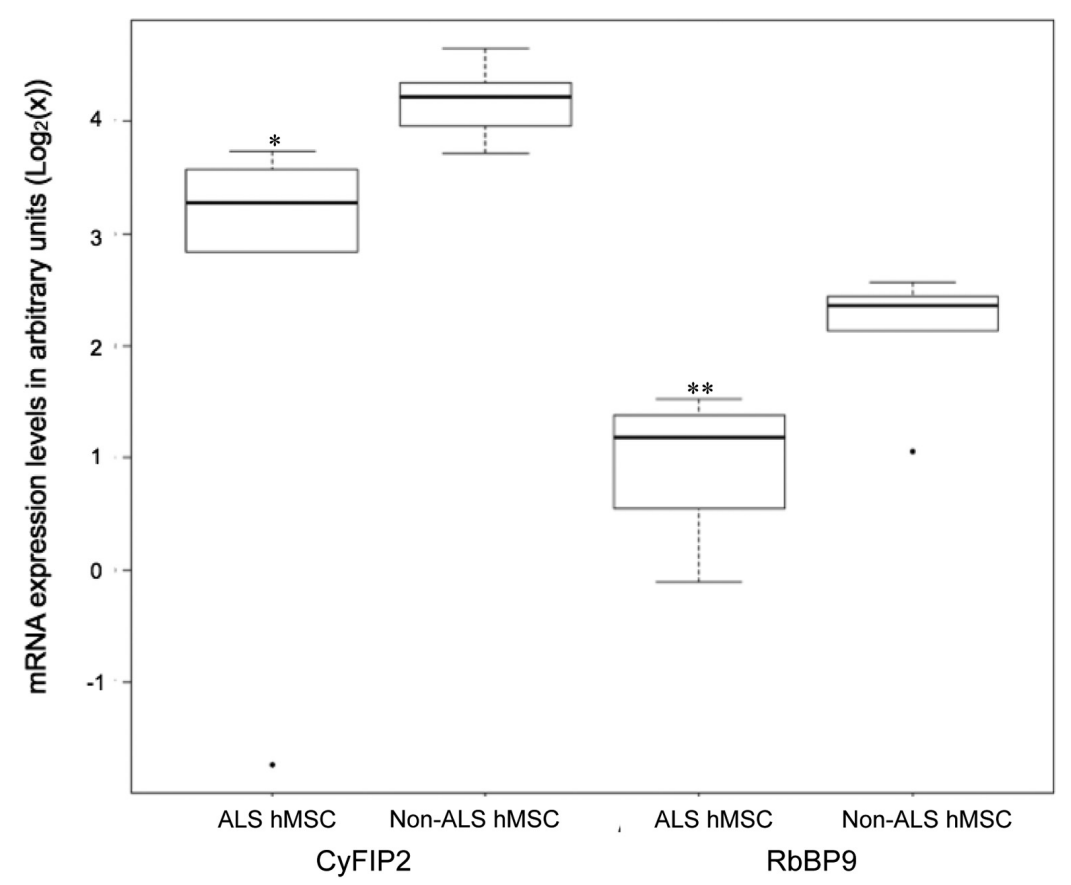

Fig. 3. Quantitative RT-PCR analysis of the expression of CyFIP2 and RbBP9 in RNA extracts of 6 ALS and 6 non-ALS hMSC. Boxplot diagram shows the qRT-PCR analysis of hMSC cultured in DMEM+10\% FBS. RLF10 served as normalizing gene for these cells. The results presented as mean \pm s.d. of relative expression of mRNA levels in arbitrary units $\left(\log _{2} X\right)$. Asterisks represent significant differences between the ALS and non-ALS obtained by T-test after square root transformation: $p=0.0028$ for CyFIP2; $p=0.0021$ for RbBP9.
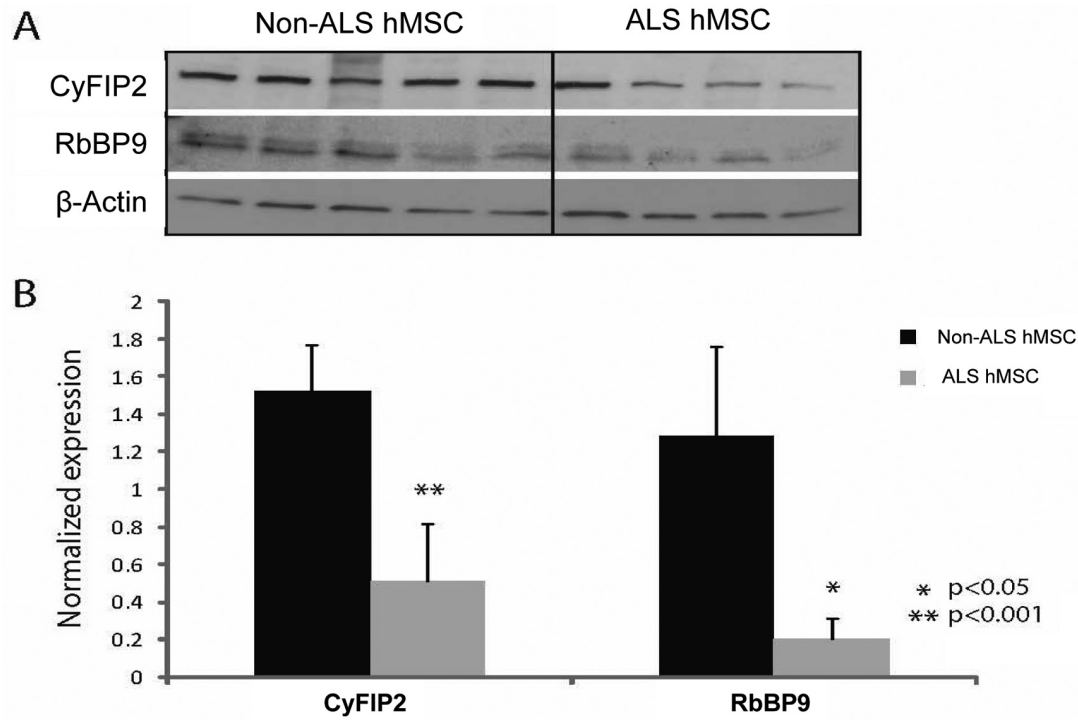

Fig. 4. Western blot analysis of the expression of CyFIP2 and RbBP9 in protein lysates of ALS non-ALS hMSC. ALS hMSC from 4 ALS patients and non-ALS hMSC from 5 control individuals at the same passage number were cultured in DMEM 10\%FBS until they reached $80 \%$ confluence. Cell lysate was extract and separated by SDS-PAGE, transferred to nitrocellulose membranes for Western blot analysis with either rabbit anti CyFIP2 or rabbit anti RbBP9 antibodies. $\beta$-actin was used as loading control for the experiments. A. Shows the ECL autoradiograms of a representative WB experiment. B. Shows the densitometry analysis of the WB results for CyFIP2 and RbBP9 respectively. The results are represented as mean values \pm s.d. Statistical analysis of the results was performed using T-test with two sample equal variance. Asterisks represent significant differences between protein levels in ALS and non-ALS cells. $p<0.05$ for RbBP9; $p<0.001$ for CyFIP2. 


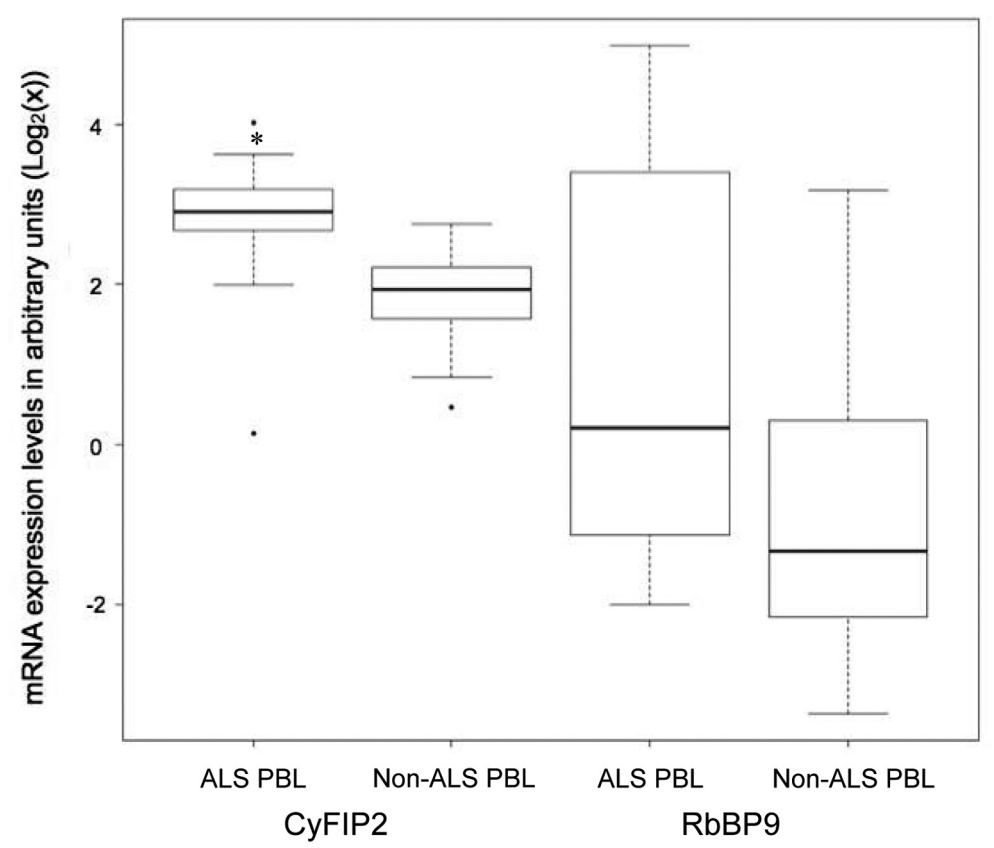

Fig. 5. Quantitative RT-PCR analysis of the expression of CyFIP2 and RbBP9 in RNA extracts from ALS and non-ALS PBL. Boxplot diagram shows the qRT-PCR analysis of PBL. RS9 served as normalizing gene for these cells. All the transcripts were detected using specific primers adequate for qRT-PCR and cDNA was synthesized by reverse transcription of RNA extracted from the PBL fraction isolated from peripheral blood samples obtained from 17 ALS and 19 non-ALS individuals. The results presented as mean \pm s.d of relative expression of mRNA levels in arbitrary units $\left(\log _{2} \mathrm{X}\right)$. Asterisks represent significant differences between the ALS and non-ALS obtained by T-test after square root transformation: $p=0.000062$ for CyFIP2; $p=0.042$ for RbBP9.

of these two genes in the hMSC and PBL derivates from several ALS individuals. It is possible that this transcriptional difference between PBL and hMSC in ALS samples may indicate abnormal transcriptional regulation of CyFIP2 and RbBP9, which expresses itself in a different manner according to the cell type. We believe that this abnormal gene expression is product of an intrinsic molecular mechanism that is deregulated in cells of ALS patients. Transcriptional regulators such as TDP43 and FUS/TLS are good candidates to test this hypothesis since both are similar DNA/RNA binding proteins that could alter RNA processing and have been directly implicated in ALS pathogenesis in familial and in sporadic cases for TDP43 [24]. These evidences support the view that CyFIP2 and RbBP9 genes are in some way abnormally regulated in cells from ALS patients that could be implicated in the mechanisms of the disease.

Interestingly, these two gene proteins could in some way be functionally related to cell cycle control and DNA damage mediated apoptosis. RbBP9 interacts with $\mathrm{Rb}$ through the LXCXE sequence and can compete with and displace E2F-1 bound to $\mathrm{Rb}$ [25]. This interaction modulates $\mathrm{Rb}$ response to radiation-induced apoptosis in vivo [26]. CyFIP2 has recently been identified as a direct target of p53 induced apoptosis [27] and in particular this molecule is expressed in the nervous system since it was found to directly interact with fragile $\mathrm{X}$ mental retardation protein (FMRP) and to be localized in mouse brain synaptosomal extracts [28]. Moreover, the Drosophila gene ortholog CyFIP is specifically expressed in the fly nervous system and mutations of this gene, affect axons and synapses that are related with biochemically and genetically interactions of dCYFIP with dFMR1 and dRac1 [27]. Recently, upregulation of CyFIP2 has been found to facilitate, via Rac 1, increased adhesion properties of activated $\mathrm{T}$ cells isolated from Multiple Sclerosis patients [28]. It will be interesting to verify if CyFIP2 or RbBP9 high expression levels in ALS PBL are implicated in some way in the immunological status of the ALS individuals. Supporting this view, we have previously shown that a thymic dysfunction observed in SOD1 mice and in ALS patients might be a co-pathological factor in ALS, regardless of the disease etiology [29].

So far, our results support the possibility that the differential expression levels of CyFIP2 and RbBP9 found in PBL of sporadic ALS patients may serve as a base for future diagnosis of sporadic ALS. To this end it is required to test the prevalence of these potential biomark- 
ers in ALS patient population at the different progressive stages of the disease. Together with this, it will be also important to test the specificity of these markers to ALS. Thus tests for differential expression of these genes in blood or bone marrow samples from patients suffering from other neurodegenerative diseases need to be made.

\section{Materials and methods}

\subsection{Cell and tissue samples}

Bone marrow samples were obtained from the iliac crest's bone marrow of six healthy donors (age 20-56 years old) and of six male ALS patients (age 43-56 years old). Peripheral blood samples were obtained from 19 healthy donors (age 21-63 years old) and of 17 ALS patients (age 30-83 years old). All volunteers in this work had signed for consent before sample donation according to the guidelines of the ethics committee of the Laniado Hospital supervised by the Israeli Health Ministry Ethics Committee conforming with The Code of Ethics of the World Medical Association (Declaration of Helsinki), printed in the British Medical Journal (18 July 1964).

\section{Isolation and culture of hMSC}

hMSC were isolated and cultured as described before [7]. hMSC at passages 3-7 were used in this study. The difference in the number of samples between ALS and non-ALS presented in Figs $1 \mathrm{~B}, 2 \mathrm{~A}$ and 4 is due to the availability of hMSC at similar passage number and growth status for the experiment. These isolated cells were routinely characterized by the expression pattern of a number of known surface markers such as $\mathrm{CD}_{105^{+}}, \mathrm{CD}^{+}{ }^{+}, \mathrm{CD}^{+} 3^{+}, \mathrm{CD} 45^{-}, \mathrm{CD} 19^{-}$and $\mathrm{CD}^{-} 4^{-}$(BioLegend, CA, USA) using FACS analysis in a similar way as previously reported [7]. The hMSC were further characterized by their ability to differentiate into adipocytes and osteocytes [20]. Cell viability or cell survival was assayed as previously reported [7], by supplementing the cells at the end of the experiment with $25 \mu \mathrm{l} / \mathrm{well}$ of a suspension made of $20 \mu \mathrm{g} / \mathrm{ml}$ Calcein-AM (Invitrogen, Oregon, USA), $2 \mu \mathrm{g} / \mathrm{ml}$ Bisbenzimide (Hoechst 33342) (Sigma, MO, USA), and $4 \mu \mathrm{g} / \mathrm{ml}$ Propidium iodide (PI) (Sigma, MO, USA) in PBS. The stained cells were incubated for 20 minutes at $37^{\circ} \mathrm{C}$ and then the proportion of viable cells was deter- mined by counting Calcein-AM positive (viable) cells out of the total Hoechst positive stained nuclei. All image acquisition was made using a Nikon DXM1200f digital camera (Nikon, Japan) mounted on an inverted fluorescence Nikon Eclipse TE2000-S microscope (Nikon, Japan) using a 10x magnification objective.

\section{Isolation of PBL from blood samples}

Blood sampleswere transferred into a $50 \mathrm{ml}$ tube containingred blood cells (RBC)lysis Buffer (89.9 g $\mathrm{NH} 4 \mathrm{Cl}, 10.0 \mathrm{~g} \mathrm{KHCO} 3$ and $2.0 \mathrm{ml} 0.5 \mathrm{M}$ EDTA dissolved in $800 \mathrm{ml}$ double distillated water $\mathrm{pH}=7.3$ ) and incubatedat room temperature for 15 minutes.Cells were centrifugedat $1400 \mathrm{rpm}$ for 10 minutes at $4^{\circ} \mathrm{C}$, The cell pellet containing Peripheral leukocytes (PBL) was resuspended in $1 \mathrm{ml}$ of RBC lysis Buffer and centrifugedat $3000 \mathrm{rpm}$ for 2 minutes in a microfuge. The cellpellet was resuspendedin $1 \mathrm{ml}$ of phosphate buffered saline (PBS) and kept on ice for RNA extraction.

\subsection{RT-PCR, quantitative realtime $-P C R$ (qRT-PCR) and RNA editing}

RNA isolation, cDNA synthesis and PCR reaction mix were performed as described previously [7]. Briefly, total RNAwas isolated from the cell pellets of hMSC cultures or of fresh PBL. The RNA (0.6-0.8 mg) was reverse-transcribed into complementary DNA (cDNA) for PCR reaction (Applied biosystem, CA, USA according to manufacturer protocol). PCR products were analyzed by electrophoresis on $2 \%$ agarose gel. The PCR primers used: GADPH forward CTTTTAACTCTGGTAAAG TGG, reverse TTTTGG CTCCCCCCTGCAAAT; CyFIP2 forward GCTGGTTCATCCCACAGACA, reverse AGGACAATGGGTCCATCCAG; RbBP9 forward GCTGCACTCATGGCTTCTCC, reverse AAAG TGGCCACAGTCAGTGAA.

Real time PCR reactions were carried out using SYBR green (Thermo Fisher Scientific Inc, CA,USA) on a Rotor Gene 6000 real-time rotator (Corbett Research). A non-template control was included for each gene. PCR reactions were performed in triplicate and standard curve points in duplicate. Absolute copy number values calculated from standard curves were normalized to a housekeeping gene ring finger protein 10 (RNF-10) for the hMSC, and ribosomal subunit 9 (RS9) for the PBL. Primers: RNF-10 forward AAGGGAGGTCACTGGTGTTG, 
reverse CTTCATCGAAGGCAGACAGA; RS9 forward CGGAGACCCTTCGAGAAATCT, reverse GCCCATACTCGCCGATCA; CyFIP2 forward CACCTGCTAAAAGTGCAGAGG, reverse TGTTCAGGATG GCAAAAACC; RbBP9 forward ACCTGGTTTCCAG TGTTTGG, reverse CAGAACTGTGGCCAATGATG. Differences between samples were analyzed for $\log 2$ transformed data by t-test, with significance set at $P<$ 0.05 .

The level of RNA editing, assessed by direct sequencing of the cDNA fragments, quantified by the Discovery Studio Gene (DSGene) 1.5 program (Accelrys Inc.). The reliability of this method was further verified by cloning of individual sequences. PCR products were ligated into pGem-T Easy (Promega, USA). After transformation of Escherichia coli, DNA was extracted using QIAprep Spin (QIAGEN, Israel), and individual plasmids were sequenced. The percentage of the edited clones was determined and compared to the DSGene quantification. This approach confirmed that the quantification was reliable even at low editing ratios of $5 \%$, representing the transcript repertoire and not background noise. The following primers were used as described previously [30]:

FANCC (Fanconi anemia, complementation group C):

5' - GGACTGGAGGAAGCACTAATACGTTGGAT GGGACTG,

3'-TGAGCCTCAGAGGTTGAGACACGTTGGAT GTGAGCC,

EXT- GAGATGGGGTTTCACC

BLCAP(Bladder cancer associated protein):

5' -ATTAGGTCGGTTCCTGCAGCACGTTGGAT

GATTAGGTCG,

3'-AGGAGGACGGGCAGCAACG TTGGATGAG

GAGGAC,

EXT-CAGCCACTGGAGGCAA

BRCA1(Breast cancer 1):

5'-TATTTTGAGATGGAGTCCAGCACG TTG

GAT GTA TTT TGA,

3' -GAAGTGCACGTTGCAGTGAGACGTTGGA

TGGA AGT CGTCGCCCAGGTTGGAGT

CYFIP2(Cytoplasmic FMR1 interacting protein 2):

5'-TTTCGGCGACATGCAGATAGACGTTGGA

TGTTTCGG,

3'-ACGTCCACTTGGACTTGTTCACGTTGGA

TGACGTCC,

EXT- CTCTTCATAGTGAGCACTGGTCT

RBBP9 (Retinoblastoma-binding protein 9):

5'-CCTTTTTTTGGCTGGGTGTGACGTTGGAT GCCTTTTTT,
3'-GCCTCAAGCAATACAACCACACGTTGGAT GGCCTCAA, EXT- GGTGGCTCATGCCTGTA

MDM4 (Mouse double minute 4, human homolog of p53-binding protein):

5'-AAAAAAATCGTGGACCGGGCACGTTGGA

TGAAA,

3'-ACCTAGGTGATCTCCCAAAGACGTTGGA

TGACCTAG,

EXT- AGTGGCTCACGCCTGTA

FLNA (Filamin A, alpha):

5'-TTCGTGGTGCCTGTGGCTTACGTTGGATG

TTCGTG,

3'-TGGGCGGTTTCTCTCGGTGACGTTGGATG

TGGGC,

EXT- TCACTGTTTCTAGCCTTC

GluR2 (Glutamate receptor subunit 2):

5'-TCTCTGGTTTTCCTTGGGTGACGTTGGAT

GTCTCTGGT,

3'-ACCACACACCTCCAACAAT

GACGTTGGATGACCACAC,

EXT-CTTGGGTGCCTTTATGC

CARD11 (Caspase recruitment domain family, member 11):

5'-AGATCACGCCACTGCACTCACGTTGGATG AGATCA,

3'-CCAGGTCAAGCTTGCCTTTTACGTTGGAT GCCAGGT,

EXT- CAGCCTGGTGACAGAGC.

\section{Western blot analysis}

Cell lysates of the hMSC cultures derived from ALS and healthy individuals (will refer as ALS and non-ALS respectively) were obtained with RIPA buffer (SigmaAldrich, MO, USA) in the presence of "Complete" protease inhibitor (Roche-Diagnostics, Basel, CH). Protein concentration was calculated by BCA protein assay kit (Pierce, IL, USA). Protein samples of $15.5 \mu \mathrm{g}$ were separated by electrophoresis through gradient 4-20\% SDS-polyacrylamide gels (Bio-Rad laboratories, Hercules, CA, USA) and transferred to nitrocellulose membranes (GE Healthcare, NJ, USA), then blocked with $5 \%(\mathrm{w} / \mathrm{v})$ non-fat milk in Tris-buffered saline/1\% tween 20 (TBS/T) for $1 \mathrm{~h}$. Primary antibodies were then added for overnight incubation at $4{ }^{\circ} \mathrm{C}$; CyFIP2 (1:1000, Proteintech group Inc.), RbBP9 (1:1000, Proteintech group Inc.), $\beta$-actin as a loading control (1:2000, MP Biomedical, OH, USA), diluted in 5\% Bovine serum albumin in TBS/T. Following three washes with TBS/T. Blots were 
probed with HRP-conjugated donkey anti-rabbit, donkey anti-mouse antibodies (Jackson Immunoresearch Laboratories, PA, USA) at 1:6000 in 5\% Bovine serum albumin in TBS/T for $1 \mathrm{~h}$ at room temperature, following three washes with TBS/T.

The detection was performed by ECL reaction (Thermo Fisher Scientific Inc, CA, USA). Protein levels were quantified by normalizing to the $\beta$-actin. Quantification of band intensities was performed by densitometry analysis using Quantity One ${ }^{\circledR}$ software (Bio-Rad laboratories). Blots were stripped using 5\% acetic acid for $20 \mathrm{~min}$; the membrane was reused with a different antibody as described above, no more than 3 times.

\section{Statistical analysis}

T-test with two sample equal variance after square root transformation was performed to establish statistical significance between the groups. A $p$ value of 0.05 or less was judged to be significant, and results were expressed as mean \pm standard deviation of average mean.

\section{Acknowledgments}

We thank Prof. Gideon Rehavi and Dr. Michal Safran for technical assistance and analysis of data in the RNA editing experiments. We thank Mr. Tal Galili from the Statistics Department at TAU for his advice in the statistical analysis of the data and to Mr. Eitan Lilo for valuable comments on the manuscript. We want to thank all the volunteers healthy or ALS individuals for their contribution to this work. This work was supported by the IsrALS foundation and by The Legacy Heritage Biomedical Program of the Israel Science Foundation (grants No. 429/09). This work is dedicated to the loving memory of Nir Tsoran and Felix Schlemowitz.

\section{References}

[1] M. Cozzolino, A. Ferri, M.T. Carri, Amyotrophic lateral sclerosis: from current developments in the laboratory to clinical implications, Antioxid Redox Signal 10 (2008) 405-443.

[2] D.W. Cleveland, J.D. Rothstein, From Charcot to Lou Gehrig: deciphering selective motor neuron death in ALS, Nat Rev Neurosci 2 (2001) 806-819.

[3] D.R. Rosen, T. Siddique, D. Patterson, D.A. Figlewicz, P. Sapp, A. Hentati, D. Donaldson, J. Goto, J.P. O'Regan, H.X. Deng et al., Mutations in $\mathrm{Cu} / \mathrm{Zn}$ superoxide dismutase gene are associated with familial amyotrophic lateral sclerosis, Nature 362 (1993) 59-62.
[4] H.X. Deng, A. Hentati, J.A. Tainer, Z. Iqbal, A. Cayabyab, W.Y. Hung, E.D. Getzoff, P. Hu, B. Herzfeldt, R.P. Roos, et al., Amyotrophic lateral sclerosis and structural defects in $\mathrm{Cu}, \mathrm{Zn}$ superoxide dismutase, Science 261 (1993) 1047-1051.

[5] L.I. Bruijn, T.M. Miller, D.W. Cleveland, Unraveling the mechanisms involved in motor neuron degeneration in ALS, Annu Rev Neurosci 27 (2004) 723-749.

[6] M.F. Pittenger, A.M. Mackay, S.C. Beck, R.K. Jaiswal, R. Douglas, J.D. Mosca, M.A. Moorman, D.W. Simonetti, S. Craig, D.R. Marshak, Multilineage potential of adult human mesenchymal stem cells, Science 284 (1999) 143-147.

[7] L.J. Solmesky, M. Abekasis, S. Bulvik, M. Weil, Bone morphogenetic protein signaling is involved in human mesenchymal stem cell survival in serum-free medium, Stem Cells Dev 18 (2009) 1283-1292.

[8] Q. Wang, J. Khillan, P. Gadue, K. Nishikura, Requirement of the RNA editing deaminase ADAR1 gene for embryonic erythropoiesis, Science 290 (2000) 1765-1768.

[9] M.J. Palladino, L.P. Keegan, M.A. O'Connell, R.A. Reenan, A-to-I pre-mRNA editing in Drosophila is primarily involved in adult nervous system function and integrity, Cell 102 (2000) 437-449.

[10] M. Higuchi, S. Maas, F.N. Single, J. Hartner, A. Rozov, N. Burnashev, D. Feldmeyer, R. Sprengel, P.H. Seeburg, Point mutation in an AMPA receptor gene rescues lethality in mice deficient in the RNA-editing enzyme ADAR2, Nature 406 (2000) 78-81.

[11] S. Maas, S. Patt, M. Schrey, A. Rich, Underediting of glutamate receptor GluR-B mRNA in malignant gliomas, Proc Natl Acad Sci U S A 98 (2001) 14687-14692.

[12] S. Maas, Y. Kawahara, K.M. Tamburro, K. Nishikura, A-to-I RNA editing and human disease, RNA Biol 3 (2006) 1-9.

[13] R. Brusa, F. Zimmermann, D.S. Koh, D. Feldmeyer, P. Gass, P.H. Seeburg, R. Sprengel, Early-onset epilepsy and postnatal lethality associated with an editing-deficient GluR-B allele in mice, Science 270 (1995) 1677-1680.

[14] Y. Kawahara, K. Ito, H. Sun, H. Aizawa, I. Kanazawa, S. Kwak, Glutamate receptors: RNA editing and death of motor neurons, Nature 427 (2004) 801.

[15] Y. Kawahara, S. Kwak, Excitotoxicity and ALS: what is unique about the AMPA receptors expressed on spinal motor neurons? Amyotroph Lateral Scler Other Motor Neuron Disord 6 (2005) 131-144.

[16] Y. Kawahara, S. Kwak, H. Sun, K. Ito, H. Hashida, H. Aizawa, S.Y. Jeong, I. Kanazawa, Human spinal motoneurons express low relative abundance of GluR2 mRNA: an implication for excitotoxicity in ALS, J Neurochem 85 (2003) 680-689.

[17] S. Kwak, Y. Kawahara, Deficient RNA editing of GluR2 and neuronal death in amyotropic lateral sclerosis, $J$ Mol Med $\mathbf{8 3}$ (2005) 110-120.

[18] S. Kwak, Y. Nishimoto, T. Yamashita, Newly identified ADAR-mediated A-to-I editing positions as a tool for ALS research, RNA Biol 5 (2008) 193-197.

[19] D.C. Colter, R. Class, C.M. Digirolamo, D.J. Prockop, Rapid expation of recycling stem cell in cultures of plastic-adherent cells from human bone marrow. Proc Natl Acad Sci USA 97 (2000) 3213-3218.

[20] M. Dominici, K.L Blanc, I. Mueller, I. Slaper-Cortenbach, F.C Marini, D.S Krause, R.J Deans, A. Keating, D.J Prockop, E.M. Horwitz, Minimal criteria for defining multipotent mesenchymal stromal cells, Cytotherapy 8 (2006) 315-317.

[21] R.R. Pochampally, J.R. Smith, J. Ylostalo, D.J. Prockop, Serum deprivation of human marrow stromal cells (hMSCs) selects for a subpopulation of early progenitor cells with 
enhanced expression of OCT-4 and other embryonic genes, Blood 103 (2004) 1647-1652.

[22] E.Y. Levanon, M. Hallegger, Y. Kinar, R. Shemesh, K. Djinovic-Carugo, G. Rechavi, M.F. Jantsch, E. Eisenberg, Evolutionarily conserved human targets of adenosine to inosine RNA editing, Nucleic Acids Res 33 (2005) 1162-1168.

[23] E.M. Riedamann, S.Schopoff, J.C. Hartner, M.F. Jantsch, Specificity of ADAR-mediated RNA editing in newly identified targets, Rna 14 (2008) 1110-1118.

[24] C.Lagier-Tourenne and D.W. Cleveland, Rethinking ALS: the FUS about TDP-43, Cell. 136 (2009) 1001-1004.

[25] J.T. Woitach, M. Zhang, C.H. Niu, S.S. Thorgeirsson, A retinoblastoma-binding protein that affects cell-cycle control and confers transforming ability, Nat Genet 19 (1998) 371374.

[26] S. Cassie, I. Koturbash, D. Hudson, M. Baker, Y. Ilnytskyy, R. Rodriguez-Juarez, E. Weber, O. Kovalchuk, Novel retinoblastoma binding protein RBBP9 modulates sex-specific radiation responses in vivo, Carcinogenesis 27 (2006) 465-474.
[27] R.S. Jackson, 2nd, Y.J. Cho, S. Stein, P. Liang, CYFIP2, a direct p53 target, is leptomycin-B sensitive, Cell Cycle 6 (2007) 95-103.

[28] A. Schenck, B. Bardoni, A. Moro, C. Bagni, J.L. Mandel, A highly conserved protein family interacting with the fragile $\mathrm{X}$ mental retardation protein (FMRP) and displaying selective interactions with FMRP-related proteins FXR1P and FXR2P, Proc Natl Acad Sci U S A 98 (2001) 8844-8849.

[29] A. Seksenyan, N. Ron-Harel, D. Azoulay, L. Cahalon, M. Cardon, P. Rogeri, M.K. Ko, M. Weil, S. Bulvik, G. Rechavi, N. Amariglio, E. Konen, M. Koronyo-Hamaoui, R. Somech, M. Schwartz, Thymic Involution in Amyotrophic Lateral Sclerosis, J Cell Mol Med 14 (2009) 2470-2482.

[30] N. Paz, E.Y. Levanon, N. Amariglio, A.B. Heimberger, Z. Ram, S. Constantini, Z.S. Barbash, K. Adamsky, M. Safran, A. Hirschberg, M. Krupsky, I. Bendov, S. Cazacu, T. Mikkelsen, C. Brodie, E. Eisenber, G. Rechavi Altered adenosine-toinosine RNA editing in human cancer, Genome Res 17 (2007) 1586-1595. 


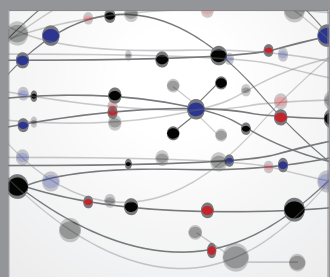

The Scientific World Journal
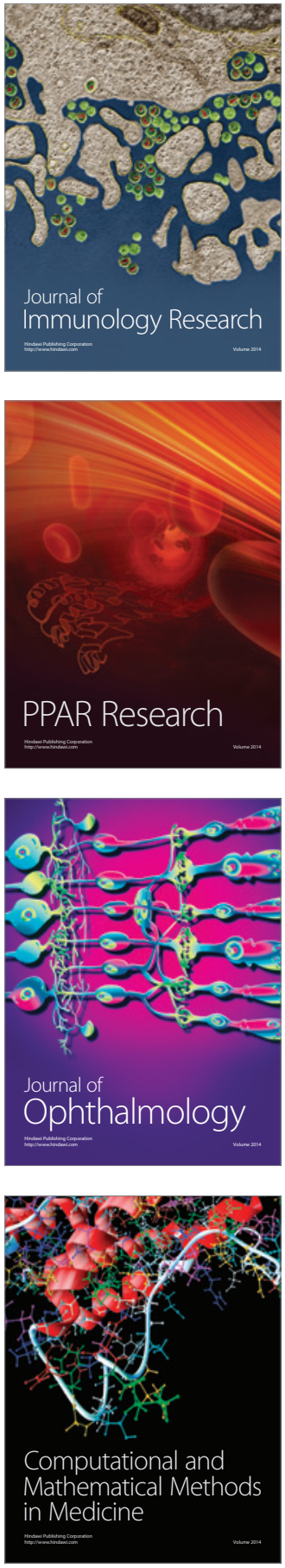

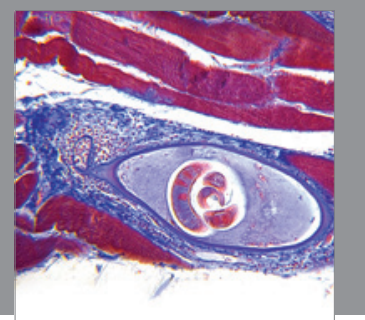

Gastroenterology

Research and Practice
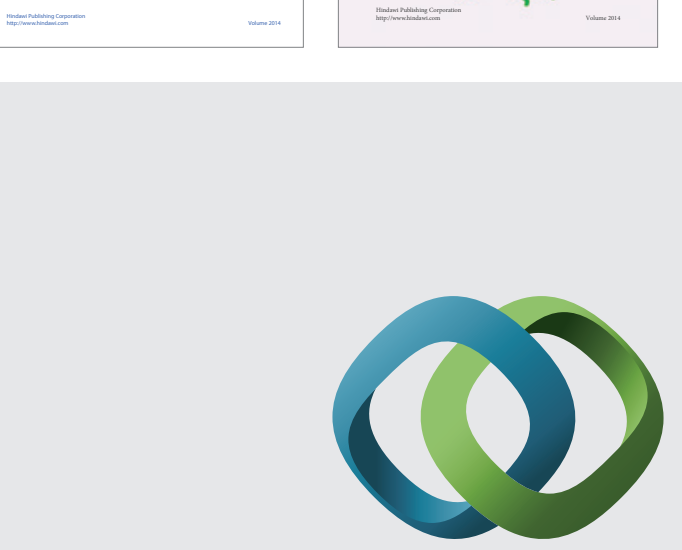

\section{Hindawi}

Submit your manuscripts at

http://www.hindawi.com
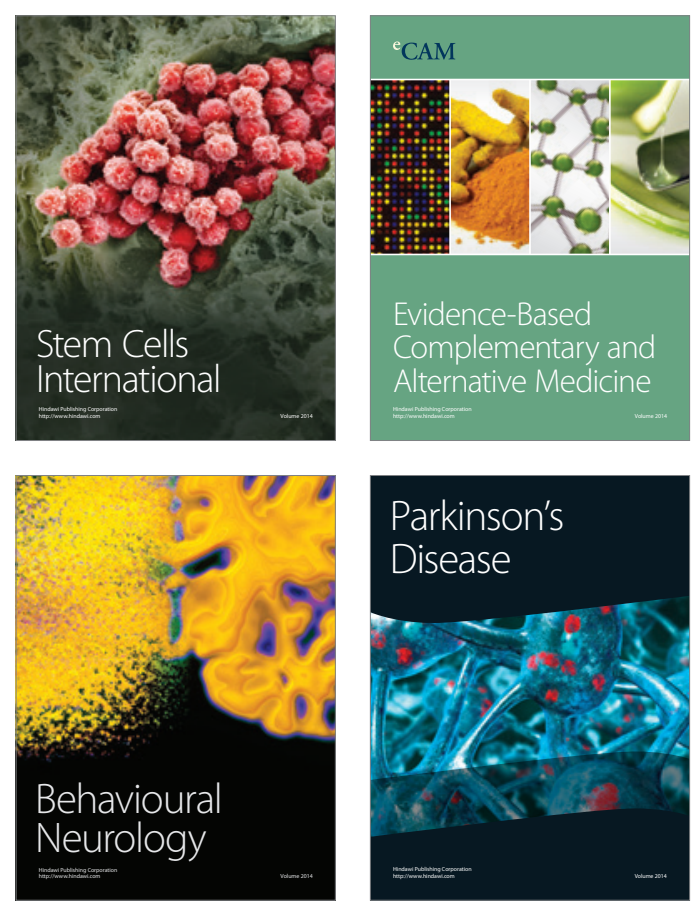

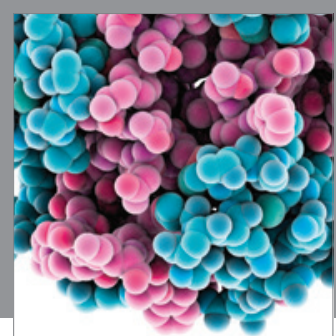

Journal of
Diabetes Research

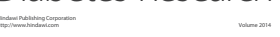

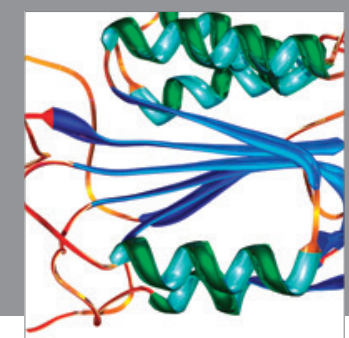

Disease Markers
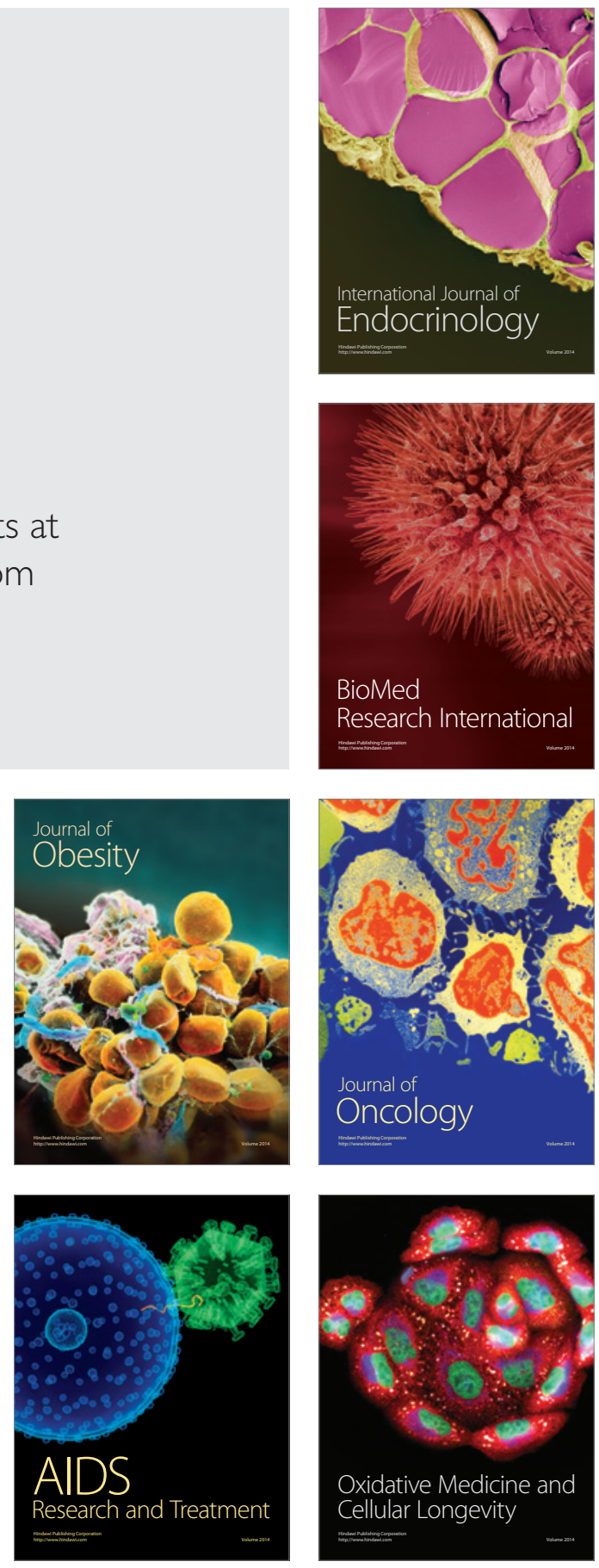